\title{
Shifted Cubic Spline Wavelets with Two Vanishing Moments on the Interval and a Splitting Algorithm
}

\author{
BORIS SHUMILOV \\ Department of Applied Mathematics \\ Tomsk State University of Architecture and Building \\ 2 Solyanaya, Tomsk, 634003 \\ RUSSIA \\ sbm@tsuab.ru
}

\begin{abstract}
This paper deals with the use of the first two vanishing moments for constructing cubic spline-wavelets orthogonal to polynomials of the first degree. A decrease in the supports of these wavelets is shown in comparison with the classical semiorthogonal wavelets. For splines with homogeneous Dirichlet boundary conditions of the second order, an algorithm of the shifted wavelet transform is obtained in the form of a solution of a tridiagonal system of linear equations with a strict diagonal dominance.
\end{abstract}

Key-Words: - $B$-splines, wavelets, implicit decomposition relations

Received: March 13, 2020. Revised: July 2, 2020. Re-revised: July 15, 2020. Accepted: July 30, 2020. Published: August 8, 2020.

\section{Introduction}

In the theory of multi-resolution analysis, wavelets form a basis of a set that fills the difference between approximating spaces on dense and sparse grids [1, p. 16]. In the classical case of approximation on uniform grids, infinitely spreading in both directions, such a basis is generated by compressions and displacements of a single wave function that has the appearance of a short or rapidly decreasing wave function (splash), which is called the wavelet. Due to compression, wavelets can identify the difference in the characteristics of a measured signal with varying degrees of details. Due to the displacement, they can analyze the properties of a signal at different points throughout the entire study interval. When analyzing non-stationary signals, the wavelets locality property provides a significant advantage over Fourier transform, which provides only global information about properties of a signal under study because the basis functions used by it (sines and cosines) have infinite supports. Since wavelets transform a system of basis functions with distributed parameters into a system with lumped parameters, such a basis is more efficient for solving problems of conditionality and convergence in numerical analysis [2, p. 478-481].

The basis for constructing wavelets is the existence of so-called scale (calibration) relations such that each basis function on a sparse grid can be expressed as a linear combination of the basis functions on a dense grid. In particular, such properties are typical for splines that are smooth functions glued together from pieces of polynomials of degree $m$ on a sequence of nested grids.
A typical property of semiorthogonal wavelets, which [3] is sometimes used as a basis for the numerical method of constructing wavelet transforms, is the fact that wavelet decomposition provides the best root-mean-square approximation of splines with the dense grid using splines on a sparse grid. This property provides an advantage in solving the problem of compressing discrete numerical information. However, this advantage is leveled due to extremely large supports $[0,2 m+1]$ of resultant spline-wavelets. Some progress in solving this problem was achieved by constructing spline-wavelets with the first $r$ moments equal to zero [4] under the condition of support length $[0,(m+r+1) / 2]$. Since semiorthogonal wavelets have $m+1$ zero moments, the idea of reducing supports of wavelets by replacing the orthogonality property for the spline space on a sparse grid with orthogonality for polynomials seems attractive. Indeed, from the speed of approximation of smooth functions $[5$, p. 52, 156] these two types of wavelets are equivalent, and orthogonality to polynomials provides a locally maximal "likeness" to a better rootmean-square approach. At the same time in statistical applications, orthogonality for polynomials of high degree is not required at all [6], in contrast, for example, to the problem of numerical differentiation [7].

In the case of cubic splines, a wavelet of the third degree $w_{3}(x)$ [8] was found for which orthogonality conditions for monomials

$$
\int_{-\infty}^{\infty} x^{k} w_{3}(x) d x=0, k=0,1
$$

are fulfilled. It turned out that this wavelet has a very 
simple structure, in particular, the support is much smaller than the support of classical semiorthogonal spline wavelet of the third degree, namely, $[0,3] \subset$ $[0,7]$. Moreover, it was found useful for constructing the basis of Riesz [9]. A similar solution [10] was found for the case of Hermite cubic spline-wavelet orthogonal to monomials of the third degree. Moreover, the original method was proposed by the author of this paper in [11, 12] for the even-odd splitting of the system of the shifted wavelet transform equations into a parallel solution of two tridiagonal systems of linear equations of double smaller order with a strict diagonal dominance. Wavelet transforms based on Hermite splines also have some disadvantages: in the problem of processing the measured information, it is necessary first to calculate the approximate values of derivatives at the nodes of the densest grid with an acceptable accuracy [13], and only then the wavelet transform algorithms can be applied. In terms of data compression, the number of wavelet coefficients, in this case, is much bigger than in methods based on $B$ splines. Therefore, Section 3 considers the pioneering idea of using even-odd splitting in the case of the shifted wavelet transform of ordinary cubic splines.

It should be noted that the method used differs considerably from the previously known fast algorithm [14, 15] of the discrete wavelet transform because it is based on the shifted wavelets with two vanishing moments instead of the interpolation problem solutions to produce statistically more smoothing (noninterpolation) solution.

The even-odd splitting of the wavelet transform matrix was independently used in [9] to prove the invertibility of the matrix; however, there was no clear indication that it can be useful for calculations in practice.

\section{Construction of cubic spline wavelets with two vanishing moments on the interval}

Let $V_{L}$ denotes a space of cubic splines of smoothness $C^{2}$ on the segment $[a, b]$ with a uniform grid consisting of the nodes $\Delta^{L}: x_{i}=a+h \cdot i, i=0,1, \ldots, 2^{L}$, $h=(b-a) / 2^{L}$, and the basis functions $N_{i}^{L}(v)=$ $\varphi_{3}(v-i) \forall i$, where $v=(x-a) / h$, with the centers in integers, are generated by means of compressions and displacements of the function of the form [16, p. 89]:

$$
\varphi_{3}(t)=\frac{1}{6} \sum_{j=0}^{4}\left(\begin{array}{l}
4 \\
j
\end{array}\right)(-1)^{j}(t-j)_{+}^{3},
$$

where $t_{+}^{n}=(\max \{t, 0\})^{n}$. Then these functions satisfy the calibration relation [1, p. 91]:

$$
\varphi_{3}(t)=\frac{1}{8} \sum_{k=0}^{4}\left(\begin{array}{l}
4 \\
k
\end{array}\right) \varphi_{3}(2 t-k) .
$$

To facilitate the construction of wavelets near the ends of a finite interval, we impose the following additional conditions on the functions: $f(a)=f^{\prime}(a)=$ $f(b)=f^{\prime}(b)=0$. The corresponding left base function has the form [14]

$$
\varphi_{b}(t)=\frac{3}{2} t_{+}^{2}-\frac{11}{12} t_{+}^{3}+\frac{3}{2}(t-1)_{+}^{3}-\frac{3}{4}(t-2)_{+}^{3},
$$

and satisfies the calibration relation

$$
\begin{aligned}
\varphi_{b}(t)=\frac{1}{4} \varphi_{b}(2 t)+\frac{11}{16} \varphi_{3}(2 t)+ & \frac{1}{2} \varphi_{3}(2 t-1)+ \\
& +\frac{1}{8} \varphi_{3}(2 t-2) .
\end{aligned}
$$

On any grid $\Delta^{L}, L \geq 2$, a resulting spline of the third degree can be represented as

$$
\begin{aligned}
S^{L}(v)= & C_{-1} \varphi_{b}(v)+\sum_{i=0}^{2^{L}-4} C_{i} \varphi_{3}(v-i)+ \\
& +C_{2^{L}-3} \varphi_{b}\left(2^{L}-v\right), 0 \leq v \leq 2^{L},
\end{aligned}
$$

where coefficients $C_{i} \forall i$ are a solution, for example, of the following interpolation problem:

$$
S^{L}(i)=f\left(x_{i}\right), i=1,2, \ldots, 2^{L}-1 .
$$

If grid $\Delta^{L-1}, L \geq 3$, is obtained from $\Delta^{L}$ by deleting each second node, then the corresponding space $V_{L-1}$ with the basis functions $N_{i}^{L-1}(v)$, whose supports are twice bigger in width and whose centers are in even nodes of the grid $\Delta^{L}$, is nested in $V_{L}$. The essence of the wavelet transform can be formulated as follows: it allows us to decompose hierarchically the given function into a series of rough approximate representations of $V_{L-1}$ and local refining details $W_{L-1}=V_{L}-V_{L-1}$.

We use for constructing a basis for $W_{L-1}$ the cubic wavelets orthogonal to all polynomials of the first degree of the following forms [8, 9]

$$
\begin{aligned}
& w_{3}(t)=-\frac{1}{2} \varphi_{3}(2 t)+\varphi_{3}(2 t-1)-\frac{1}{2} \varphi_{3}(2 t-2), \\
& w_{b}(t)=\varphi_{b}(2 t)-1.35 \varphi_{3}(2 t)+0.6 \varphi_{3}(2 t-1) .(4)
\end{aligned}
$$

They have two zero moments

$\int_{-\infty}^{\infty} x^{k} w_{3}(x) d x=\int_{-\infty}^{\infty} x^{k} w_{b}(x) d x=0, k=0,1$,

in addition, these wavelets have the supports reduced:

$$
\operatorname{supp} w_{3}=[0,3], \operatorname{supp} w_{b}=[0,2.5] .
$$




\subsection{Construction of the defining system of the shifted wavelet transform equations}

For further reasoning, it is convenient to write the basis spline functions in the form of a single row matrix,

$$
\begin{gathered}
\varphi^{L}(\cdot)=\left[\varphi_{b}(\cdot), \varphi_{3}(\cdot), \varphi_{3}(\cdot-1), \ldots\right. \\
\left.\quad \ldots, \varphi_{3}\left(\cdot-2^{L}+4\right), \varphi_{b}\left(2^{L}-\cdot\right)\right]
\end{gathered}
$$

and to arrange the spline coefficients in the form of a vector, $C^{L}=\left[C_{-1}, C_{0}, \ldots, C_{2^{L}-3}\right]^{T}$. Then formula (3) is rewritten as $S^{L}(\cdot)=\varphi^{L}(\cdot) C^{L}$.

Similarly, we can write the basis shifted wavelet functions, with the centers in odd integers instead of the centers in even integers for the case of classical semiorthogonal spline wavelets, in the form of a row matrix as

$$
\begin{gathered}
\psi^{L}(\cdot)=\left[w_{b}(\cdot), w_{3}(\cdot), w_{3}(\cdot-1), \ldots\right. \\
\left.\ldots, w_{3}\left(\cdot-2^{L}+3\right), w_{b}\left(2^{L}-\cdot\right)\right] .
\end{gathered}
$$

The corresponding coefficients of the decomposition wavelets at the level $L$ are assembled into a vector, $D^{L}=\left[D_{-1}, D_{0}, \ldots, D_{2^{L}-2}\right]^{T}$. Meanwhile, the condition of the complementarity of sizes of the resultant spaces is $\operatorname{Dim}\left(V_{L}\right)=\operatorname{Dim}\left(V_{L-1}\right)+\operatorname{Dim}\left(W_{L-1}\right)$. Then, for the level of the expansion of $L-1$, the functions $\varphi^{L-1}(\cdot)$ and $\psi^{L-1}(\cdot)$ can be written as linear combinations of the functions $\varphi^{L}(\cdot)$ :

$$
\varphi^{L-1}(\cdot)=\varphi^{L}(\cdot) P^{L} \text { and } \psi^{L-1}(\cdot)=\varphi^{L}(\cdot) Q^{L},
$$

where the columns of the matrix $P^{L}$ are composed of the relation coefficients (11) and (2) since each wide basis function within the approximation interval can be constructed from five narrow basis functions, each wide basis function at the ends of the interval can be constructed from four narrow basis functions while the elements of the columns of the matrix $Q^{L}$ are composed of the relation coefficients (4).

Consequently, the following equalities hold:

$$
\begin{aligned}
\varphi^{L}(\cdot) C^{L} & =\varphi^{L-1}(\cdot) C^{L-1}+\psi^{L-1}(\cdot) D^{L-1}= \\
& =\varphi^{L}(\cdot) P^{L} C^{L-1}+\varphi^{L}(\cdot) Q^{L} D^{L-1} .
\end{aligned}
$$

Thus, the process of obtaining $C^{L}$ from $C^{L-1}$ and $D^{L-1}$ can be written in the form

$$
C^{L}=P^{L} C^{L-1}+Q^{L} D^{L-1}
$$

or, using the notation for block matrices,

$$
C^{L}=\left[P^{L} \mid Q^{L}\right]\left[\frac{C^{L-1}}{D^{L-1}}\right] .
$$

The following example shows how to get three basis spline functions from $V_{2}$ and four base wavelets from $W_{2}$ using seven basis functions from $V_{3}$ :

$$
\begin{gathered}
{\left[P^{3} \mid Q^{3}\right]=} \\
{\left[\begin{array}{ccc|cccc}
\frac{1}{4} & 0 & 0 & 1 & 0 & 0 & 0 \\
\frac{11}{16} & \frac{1}{8} & 0 & -1.35 & -\frac{1}{2} & 0 & 0 \\
\frac{1}{2} & \frac{1}{2} & 0 & 0.6 & 1 & 0 & 0 \\
\frac{1}{8} & \frac{3}{4} & \frac{1}{8} & 0 & -\frac{1}{2} & -\frac{1}{2} & 0 \\
0 & \frac{1}{2} & \frac{1}{2} & 0 & 0 & 1 & 0.6 \\
0 & \frac{1}{8} & \frac{11}{16} & 0 & 0 & -\frac{1}{2} & -1.35 \\
0 & 0 & \frac{1}{4} & 0 & 0 & 0 & 1
\end{array}\right] .}
\end{gathered}
$$

Define the block matrix that inverse to the matrix $\left[P^{L} \mid Q^{L}\right]$ :

$$
\left[\frac{A^{L}}{B^{L}}\right]=\left[P^{L} \mid Q^{L}\right]^{-1} .
$$

Then the process of creating a version with a coarse resolution, $C^{L-1}$, characterized by fewer coefficients, can be expressed by the matrix equality

$$
C^{L-1}=A^{L} C^{L},
$$

where $A^{L}$ is a matrix of dimension $\left(2^{L-1}-1\right) \times$ $\left(2^{L}-1\right)$. In this case, the lost parts are collected in another vector $D^{L-1}$, defined by the expression

$$
D^{L-1}=B^{L} C^{L},
$$

where $B^{L}$ is a matrix of dimension $2^{L-1} \times\left(2^{L}-1\right)$. Matrices $A^{L}$ and $B^{L}$ are called analysis filters, and matrices $P^{L}$ and $Q^{L}$ are called synthesis filters [2, p. 198], [17, p. 95].

The procedure for splitting coefficients $C^{L}$ into a coarse version $C^{L-1}$ and refining coefficients $D^{L-1}$ can be applied recursively to this part $C^{L-1}$ itself. Consequently, initial coefficients can be represented as a hierarchy of rough versions with resolutions $C^{0}, C^{1}, \ldots, C^{L-1}$ and refinements $D^{0}, D^{1}, \ldots, D^{L-1}$. A similar recursive process is called a filter block [17, p. 95]. Moreover, by values of wavelet coefficients $D^{j}, j=0,1, \ldots, L-1$, one can judge a significance of corresponding refinement details. Insignificant details are removed in order to compress information. Finally, coefficients $C^{L}$ can be reconstructed from a sequence $C^{0}, D^{0}, D^{1}, \ldots, D^{L-1}$.

Unfortunately, as is easy to see, the matrices that inverse to $\left[P^{L} \mid Q^{L}\right]$ lose their sparse structure. This corresponds to how, in the classical theory of wavelets, the explicit expansion of the basis functions 
of a spline space on a dense grid contains an infinite sum of the basis functions of a spline space on a thinned grid and wavelets [1, p. 142, 199]. The essence of the approach proposed in [17, p. 96] for such cases is that $C^{L-1}$ and $D^{L-1}$ can be calculated from $C^{L}$ by solving the system of linear equations (6). Moreover, they proposed to remake the matrix $\left[P^{L} \mid Q^{L}\right]$ into the tape matrix by simply changing the order of the unknowns so that the columns of the matrices $P^{L}$ and $Q^{L}$ were interleaved.

In our case, we can transform the matrix $\left[P^{L} \mid Q^{L}\right]$ into a five-diagonal form, for example,

$$
\begin{gathered}
{\left[P^{3} \mid Q^{3}\right] \Rightarrow} \\
{\left[\begin{array}{ccccccc}
1 & \frac{1}{4} & & & & \\
-1.35 & \frac{11}{16} & -\frac{1}{2} & \frac{1}{8} & & & \\
0.6 & \frac{1}{2} & 1 & \frac{1}{2} & & & \\
& \frac{1}{8} & -\frac{1}{2} & \frac{3}{4} & -\frac{1}{2} & \frac{1}{8} & \\
& & & \frac{1}{2} & 1 & \frac{1}{2} & 0.6 \\
& & & \frac{1}{8} & -\frac{1}{2} & \frac{11}{16} & -1.35 \\
& & & & & \frac{1}{4} & 1
\end{array}\right] .}
\end{gathered}
$$

Hereinafter the empty positions of the matrices are zero. Thus, the operation of wavelet decomposition can be performed without explicitly presenting and using the filters block. Nevertheless, although the solvability of the resulting system is guaranteed by the linear independence of the basis functions, the question of its good conditionality remains open. As can be seen from the above example, the derived system of equations does not have a diagonal dominance, which can complicate the wavelet analysis of big size data.

\section{Splitting algorithm}

Let for resolution levels $L$, the matrix $G^{L}$ of size $\left(2^{L}-1\right) \times\left(2^{L}-1\right)$ has the form

$\left[\begin{array}{ccccccccc}8 & -4 & -\frac{296}{3} & 0 & & & & & \\ 0 & 9 & 0 & 0 & 0 & & & & \\ 12 & 0 & 2 & 3 & 3 & 0 & & & \\ 0 & 0 & 0 & 2 & 0 & 0 & 0 & & \\ 0 & 0 & 54 & 3 & 10 & 3 & 3 & 0 & \\ 0 & 0 & 0 & 0 & 0 & 2 & 0 & 0 & \\ 0 & 0 & 0 & 0 & 3 & 3 & 10 & 3 & 3 \\ & & & \ddots & \ddots & \ddots & \ddots & \ddots & \\ & 0 & 3 & 3 & 10 & 3 & 54 & 0 & 0 \\ & & 0 & 0 & 0 & 2 & 0 & 0 & 0 \\ & & & 0 & 3 & 3 & 2 & 0 & 12 \\ & & & & 0 & 0 & 0 & 9 & 0 \\ & & & & & 0 & -\frac{296}{3} & -4 & 8\end{array}\right]$,

where diagonal points mean that the preceding two rows are repeated the appropriate number of times, each time moving two positions to the right, while the matrix $R^{L}$ is compound from two blocks according to $2^{L-1}-1$ basis spline functions of $V_{L-1}$ and $2^{L-1}$ base wavelets of $W_{L-1}$ :

$\left[\begin{array}{ccccccccc}12 & 4 & -108 & 0 & 0 & 0 & & & \\ 0 & 0 & 72 & 4 & 4 & 0 & 0 & & \\ 0 & 0 & 0 & 0 & 4 & 4 & 4 & 0 & \\ & & & & \ddots & \ddots & \ddots & & \\ & & 0 & 0 & 4 & 4 & 72 & 0 & 0 \\ & & & 0 & 0 & 0 & -108 & 4 & 12 \\ \hline & & & & & & & & \\ 5 & -5 & -\frac{215}{3} & 0 & 0 & 0 & & & \\ 3 & 1 & 63 & 1 & 1 & 0 & 0 & & \\ 0 & 0 & 18 & 1 & 6 & 1 & 1 & 0 & \\ 0 & 0 & 0 & 0 & 1 & 1 & 6 & 1 & 1 \\ & & & \ddots & \ddots & \ddots & \ddots & \ddots & \\ & 0 & 1 & 1 & 6 & 1 & 18 & 0 & 0 \\ & & & 0 & 1 & 1 & 63 & 1 & 3 \\ & & & & 0 & 0 & -\frac{215}{3} & -5 & 5\end{array}\right]$,

where diagonal points mean that the preceding row is repeated the corresponding number of times while going right on one position.

We propose to perform calculations based on our previously developed procedure [18, 19] of the evenodd splitting of shifted wavelet decomposition of the form (5), connecting the basis functions of the space of splines on a dense grid, the basis functions on a sparse grid, and shifted wavelets by finite implicit relations of the expansion with three non-empty diagonals and strict diagonal dominance. For the type of wavelets presented above, similar results are valid, which, using the notations above, can be represented in the following form.

Lemma 1 The basis functions of cubic spline space on a dense grid, the basis functions on a sparse grid, and shifted wavelets satisfy the matrix equality

$$
\varphi^{L}(\cdot) G^{L}=\left[\varphi^{L-1}(\cdot) \mid \psi^{L-1}(\cdot)\right] R^{L}, L \geq 4 .
$$

Proof. According to the construction, at the left end of the interval $[a, b]$ inside the support of the first two wide basis functions, 3 wavelets and 6 narrow basis functions overlap. Therefore, using the calibration relations introduced earlier, we can write the following finite implicit decomposition relation:

$$
\begin{gathered}
C_{-1}^{L} \varphi_{b}(v)+\sum_{i=0}^{4} C_{i}^{L} \varphi_{3}(v-i)= \\
=C_{-1}^{L-1}\left(\frac{1}{4} \varphi_{b}(v)+\frac{11}{16} \varphi_{3}(v)+\frac{1}{2} \varphi_{3}(v-1)+\right. \\
\left.+\frac{1}{8} \varphi_{3}(v-2)\right)+ \\
+C_{0}^{L-1}\left(\frac{1}{8} \sum_{k=0}^{4}\left(\begin{array}{l}
4 \\
k
\end{array}\right) \varphi_{3}(v-k)\right)+
\end{gathered}
$$




$$
\begin{gathered}
+D_{-1}^{L-1}\left(\varphi_{b}(v)-1.35 \varphi_{3}(v)+0.6 \varphi_{3}(v-1)\right)+ \\
+\sum_{j=0}^{1} D_{j}^{L-1}\left(-\frac{1}{2} \varphi_{3}(v-j)+\varphi_{3}(v-j-1)-\right. \\
\left.-\frac{1}{2} \varphi_{3}(v-j-2)\right), 0 \leq v \leq 8 .
\end{gathered}
$$

Since cubic splines are presented on both sides of the equality (9), for their coincidence, the equality of the corresponding expansion coefficients on the basis of $B$-splines on a dense grid is sufficient. Then, to calculate the uncertain coefficients in the relation (9), we have, respectively, the following equations for the numbers $i=-1,0, \ldots, 4$

$$
\begin{aligned}
C_{-1}^{L} & =\frac{1}{4} C_{-1}^{L-1}+D_{-1}^{L-1} \\
C_{0}^{L} & =\frac{11}{16} C_{-1}^{L-1}+\frac{1}{8} C_{0}^{L-1}-1.35 D_{-1}^{L-1}-\frac{1}{2} D_{0}^{L-1} \\
C_{1}^{L} & =\frac{1}{2} C_{-1}^{L-1}+\frac{1}{2} C_{0}^{L-1}+0.6 D_{-1}^{L-1}+D_{0}^{L-1} \\
C_{2}^{L} & =\frac{1}{8} C_{-1}^{L-1}+\frac{3}{4} C_{0}^{L-1}-\frac{1}{2} D_{0}^{L-1}-\frac{1}{2} D_{1}^{L-1} \\
C_{3}^{L} & =\frac{1}{2} C_{0}^{L-1}+D_{1}^{L-1} \\
C_{4}^{L} & =\frac{1}{8} C_{0}^{L-1}-\frac{1}{2} D_{1}^{L-1}
\end{aligned}
$$

We will try to find a decomposition relation relating to the expansion coefficients for odd numbers (the case when $C_{0}^{L}=C_{2}^{L}=C_{4}^{L}=0$ ). It is easy to verify that the system obtained has a nontrivial solution for which:

$$
C_{-1}^{L}=-\frac{296}{3}, C_{1}^{L}=2, C_{3}^{L}=54 .
$$

Wherein $C_{-1}^{L-1}=-108, C_{0}^{L-1}=72$, and $D_{-1}^{L-1}=-\frac{215}{3}, D_{0}^{L-1}=63, D_{1}^{L-1}=18$.

There is another non-trivial solution that binds the expansion coefficients for the numbers -1 and 1 :

$$
C_{-1}^{L}=8, C_{1}^{L}=12, C_{3}^{L}=0 .
$$

Wherein $C_{-1}^{L-1}=12, C_{0}^{L-1}=0$, and $D_{-1}^{L-1}=$ $5, D_{0}^{L-1}=3, D_{1}^{L-1}=0$.

We will try to find a decomposition relation relating to the expansion coefficients for three neighboring numbers. In the case when $C_{-1}^{L}=C_{0}^{L}=C_{4}^{L}=0$, we get:

$$
C_{1}^{L}=C_{3}^{L}=3, C_{2}^{L}=2 .
$$

Wherein $C_{-1}^{L-1}=0, C_{0}^{L-1}=4$, and $D_{-1}^{L-1}=$ $0, D_{0}^{L-1}=D_{1}^{L-1}=1$.

There is another non-trivial solution that binds the expansion coefficients for the numbers -1 and 0 :

$$
C_{-1}^{L}=-4, C_{0}^{L}=9, C_{1}^{L}=C_{2}^{L}=C_{3}^{L}=0 .
$$

Wherein $C_{-1}^{L-1}=4, C_{0}^{L-1}=0$, and $D_{-1}^{L-1}=$ $-5, D_{0}^{L-1}=1, D_{1}^{L-1}=0$.

According to the construction, inside the interval, $[a, b]$, in the support of every two wide basis functions, 3 wavelets, and 7 narrow basis functions overlap. For example, on the segment $\left[x_{0}, x_{10}\right]$, we can write the following finite implicit decomposition relation:

$$
\begin{gathered}
\sum_{i=0}^{6} C_{i}^{L} \varphi_{3}(v-i)= \\
=\sum_{j=0}^{1} C_{j}^{L-1}\left(\frac{1}{8} \sum_{k=0}^{4}\left(\begin{array}{l}
4 \\
k
\end{array}\right) \varphi_{3}(v-j-k)\right)+ \\
+\sum_{j=0}^{2} D_{j}^{L-1}\left(-\frac{1}{2} \varphi_{3}(v-j)+\varphi_{3}(v-j-1)-\right. \\
\left.-\frac{1}{2} \varphi_{3}(v-j-2)\right), 0 \leq v \leq 10 .
\end{gathered}
$$

Since cubic splines are presented on both sides of the equality (10), for their coincidence, the equality of the corresponding expansion coefficients on the basis of $B$-splines on a dense grid is sufficient. Then, to calculate the uncertain coefficients in the relation (10), we have, respectively, the following equations for the numbers $i=0,1, \ldots, 6$

$$
\begin{aligned}
C_{0}^{L} & =\frac{1}{8} C_{0}^{L-1}-\frac{1}{2} D_{0}^{L-1}, \\
C_{1}^{L} & =\frac{1}{2} C_{0}^{L-1}+D_{0}^{L-1}, \\
C_{2}^{L} & =\frac{3}{4} C_{0}^{L-1}+\frac{1}{8} C_{1}^{L-1}-\frac{1}{2} D_{0}^{L-1}-\frac{1}{2} D_{1}^{L-1}, \\
C_{3}^{L} & =\frac{1}{2} C_{0}^{L-1}+\frac{1}{2} C_{1}^{L-1}+D_{1}^{L-1}, \\
C_{4}^{L} & =\frac{1}{8} C_{0}^{L-1}+\frac{3}{4} C_{1}^{L-1}-\frac{1}{2} D_{1}^{L-1}-\frac{1}{2} D_{2}^{L-1}, \\
C_{5}^{L} & =\frac{1}{2} C_{1}^{L-1}+D_{2}^{L-1}, \\
C_{6}^{L} & =\frac{1}{8} C_{1}^{L-1}-\frac{1}{2} D_{2}^{L-1} .
\end{aligned}
$$

For $C_{3}^{L}=10$, we try to find a decomposition relation relating to the expansion coefficients for odd numbers (the case when $C_{0}^{L}=C_{2}^{L}=C_{4}^{L}=C_{6}^{L}=$ $0)$. It is easy to verify that the system obtained has a unique nontrivial solution for which

$$
C_{1}^{L}=C_{5}^{L}=3 .
$$

Wherein $C_{0}^{L-1}=C_{1}^{L-1}=4$, and $D_{0}^{L-1}=$ $D_{2}^{L-1}=1, D_{1}^{L-1}=6$.

There are two more solutions linking the expansion coefficients for three neighboring nodes. In the case when $C_{0}^{L}=C_{4}^{L}=C_{5}^{L}=C_{6}^{L}=0$, the solution coincides with one of the solutions obtained above: $C_{1}^{L}=C_{3}^{L}=3, C_{2}^{L}=2, C_{0}^{L-1}=4, C_{1}^{L-1}=0$, and $D_{0}^{L-1}=D_{1}^{L-1}=1, D_{-1}^{L-1}=0$. 
In the case when $C_{0}^{L}=C_{1}^{L}=C_{2}^{L}=C_{6}^{L}=0$, the solution is symmetric:

$$
C_{3}^{L}=C_{5}^{L}=3, C_{4}^{L}=2 .
$$

Wherein $C_{0}^{L-1}=0, C_{1}^{L-1}=4$, and $D_{1}^{L-1}=$ $D_{2}^{L-1}=1, D_{0}^{L-1}=0$.

The same solutions hold for all subsequent pairs of wide basis functions.

At the right end of the interval $[a, b]$, the solutions mirror the solutions obtained above, i.e. expansion coefficients for odd numbers (the case when $C_{2^{L}-4}^{L}=$ $C_{2^{L}-6}^{L}=C_{2^{L}-8}^{L}=0$ ) are equal to:

$$
C_{2^{L}-3}^{L}=-\frac{296}{3}, C_{2^{L}-5}^{L}=2, C_{2^{L}-7}^{L}=54 .
$$

Wherein $C_{2^{L-1}-3}^{L-1}=-108, C_{2^{L-1}-4}^{L-1}=72$, and $D_{2^{L-1}-2}^{L-1}=-\frac{215}{3}, D_{2^{L-1}-3}^{L-1}=63, D_{2^{L-1}-4}^{L-1}=18$.

There is another non-trivial solution that binds the expansion coefficients for the numbers $2^{L}-3$ and $2^{L}-5$ :

$$
C_{2^{L}-3}^{L}=8, C_{2^{L}-5}^{L}=12, C_{2^{L}-5}^{L}=0 .
$$

Wherein $C_{2^{L-1}-3}^{L-1}=12, C_{2^{L-1}-4}^{L-1}=0$, and $D_{2^{L-1}-2}^{L-1}=5, D_{2^{L-1}-3}^{L-1}=3, D_{2^{L-1}-4}^{L-1}=0$.

In the case when $C_{2^{L}-3}^{L}=C_{2^{L}-4}^{L}=C_{2^{L}-8}^{L}=0$, the solution coincides with one of the solutions obtained on the penultimate pair of broad basis functions for three adjacent numbers: $C_{2^{L}-5}^{L}=C_{2^{L}-7}^{L}=$ $3, C_{2^{L}-6}^{L}=2, C_{2^{L-1}-3}^{L-1}=0, C_{2^{L-1}-4}^{L-1}=4$, and $D_{2^{L-1}-3}^{L-1}=D_{2^{L-1}-4}^{L-1}=1, D_{2^{L-1}-2}^{L-1}=0$.

And there is another non-trivial solution that links the expansion coefficients for the numbers $2^{L}-3$ and $2^{L}-4$ :

$$
\begin{gathered}
C_{2^{L}-3}^{L}=-4, C_{2^{L}-4}^{L}=9, \\
C_{2^{L}-5}^{L}=C_{2^{L}-6}^{L}=C_{2^{L}-7}^{L}=0 .
\end{gathered}
$$

Wherein $C_{2^{L-1}-3}^{L-1}=4, C_{2^{L-1}-4}^{L-1}=0$, and $D_{2^{L-1}-2}^{L-1}=-5, D_{2^{L-1}-3}^{L-1}=1, D_{2^{L-1}-4}^{L-1}=0$.

The coefficients of the left and right sides of the obtained expansions comprise the corresponding blocks of the matrices $G^{L}$ and $R^{L}$, which were introduced in the conditions of Lemma 1.

Corollary 1 For any resolution level $L \geq 4$, the shifted wavelet decomposition matrix of cubic splines satisfies the equation

$$
\left[P^{L} \mid Q^{L}\right] R^{L}=G^{L} .
$$

Proof. It follows from the definition of the shifted wavelet decomposition matrix that

$$
\left[\varphi^{L-1}(\cdot) \mid \psi^{L-1}(\cdot)\right]=\varphi^{L}(\cdot)\left[P^{L} \mid Q^{L}\right] .
$$

Substituting the obtained expansion into the equality (8) and taking into account the linear independence of the basis splines, we arrive at the statement of Corollary 1.

Proposition 1 For $L=3$ equation (11) is verified by direct calculation using the example (7) and definitions

$$
\begin{aligned}
G^{3} & =\left[\begin{array}{ccccccc}
8 & -4 & -\frac{296}{3} & 0 & 0 & 0 & 0 \\
0 & 9 & 0 & 0 & 0 & 0 & 0 \\
12 & 0 & 2 & 12 & 54 & 0 & 0 \\
0 & 0 & 0 & 8 & 0 & 0 & 0 \\
0 & 0 & 54 & 12 & 2 & 0 & 12 \\
0 & 0 & 0 & 0 & 0 & 9 & 0 \\
0 & 0 & 0 & 0 & -\frac{296}{3} & -4 & 8
\end{array}\right], \\
R^{3} & =\left[\begin{array}{ccccccc}
12 & 4 & -108 & 0 & 0 & 0 & 0 \\
0 & 0 & 72 & 16 & 72 & 0 & 0 \\
0 & 0 & 0 & 0 & -108 & 4 & 12 \\
\hline 5 & -5 & -\frac{215}{3} & 0 & 0 & 0 & 0 \\
3 & 1 & 63 & 8 & 18 & 0 & 0 \\
0 & 0 & 18 & 4 & 63 & 1 & 3 \\
0 & 0 & 0 & 0 & -\frac{215}{3} & -5 & 5
\end{array}\right] .
\end{aligned}
$$

After that, the solution of the system of equations (6) can be written in a matrix form as:

$$
\left[\frac{C^{L-1}}{D^{L-1}}\right]=\left[P^{L} \mid Q^{L}\right]^{-1} C^{L}=R^{L}\left(G^{L}\right)^{-1} C^{L} .
$$

So, instead of just solving the system of the form (6) we can solve the system

$$
G^{L} \Xi^{L}=C^{L}
$$

according to some values $\Xi^{L}$ and after that simply evaluate the values $C^{L-1}$ and $D^{L-1}$ with use of linear transform $\left[\frac{C^{L-1}}{D^{L-1}}\right]=R^{L} \Xi^{L}$.

Nevertheless, we still need to split the system (12) into even and odd nodes and adapt the result to provide a strict diagonal dominance to reduce the algorithm to the solution of a tridiagonal system of equations that is preferable for parallelization and stability of computation. We will achieve this goal by combining the tridiagonal sweep method with the incomplete reduction method studied in [20, p. 198].

The following statement describes formally the sequence of calculation of shifted wavelet coefficients based on the known spline expansion coefficients on an arbitrary grid $\Delta^{L}, L \geq 3$. 
Theorem 1 Suppose that the values $\Xi^{L}=$ $\left[\xi_{-1}, \ldots, \xi_{2^{L}-3}\right]^{T}$ are computed from the solution of the equations:

$$
\begin{aligned}
\xi_{i} & =\frac{1}{9} C_{i}, i=0,2^{L}-4 \\
\xi_{i} & =\frac{1}{2} C_{i}, i=2,4, \ldots, 2^{L}-6 ;
\end{aligned}
$$

a) $L>3$ :

$$
\begin{array}{rc}
446 \xi_{3}+150 \xi_{5} & =27 C_{-1}+12 C_{0}- \\
-18 C_{1}-48 C_{2} & +50 C_{3}-75 C_{4} \\
6 \xi_{i-2}+20 \xi_{i}+6 \xi_{i+2} & =2 C_{i}-3 C_{i-1}-3 C_{i+1}, \\
& i=5,7, \ldots, 2^{L}-9 \\
150 \xi_{i-2}+446 \xi_{i} & =27 C_{i+4}+12 C_{i+3}- \\
& +50 C_{i}-75 C_{i-1}, \\
-18 C_{i+2}-48 C_{i+1} & i=2^{L}-7 ; \\
300 \xi_{1} & =2 C_{1}-3 C_{-1}-3 C_{2}- \\
-\frac{4}{3} C_{0}-6 \xi_{3} & \\
300 \xi_{2^{L}-5}=2 C_{2^{L}-5}-3 C_{2^{L}-3}-3 C_{2^{L}-6}- \\
-\frac{4}{3} C_{2^{L}-4}-6 \xi_{2^{L}-7} \\
12 \xi_{-1}=C_{1}-\frac{3}{2} C_{2}-2 \xi_{1}-3 \xi_{3} \\
12 \xi_{2^{L}-3}=C_{2^{L}-5}-\frac{3}{2} C_{2^{L}-6}-2 \xi_{2^{L}-5}- \\
-3 \xi_{2^{L}-7} ;
\end{array}
$$

b) $L=3$ :

$$
\begin{aligned}
300 \xi_{1}+108 \xi_{3} & =2 C_{1}-3 C_{-1}-3 C_{2}-\frac{4}{3} C_{0}, \\
108 \xi_{1}+300 \xi_{3} & =2 C_{3}-3 C_{5}-3 C_{2}-\frac{4}{3} C_{4}(16) \\
12 \xi_{-1} & =C_{1}-\frac{3}{2} C_{2}-2 \xi_{1}-54 \xi_{3}, \\
12 \xi_{5} & =C_{3}-\frac{3}{2} C_{2}-2 \xi_{3}-54 \xi_{1} \cdot(17)
\end{aligned}
$$

Then the vector of spline-coefficients of size $\left(2^{L-1}-1\right)$ on the sparse grid $\Delta^{L-1}$ is the result of multiplication of the matrix $A^{L}$ of size $\left(2^{L-1}-1\right) \times$ $\left(2^{L}-1\right)$ by the vector $\Xi^{L}$ of size $\left(2^{L}-1\right)$, while the vector of shifted wavelet coefficients of size $2^{L-1}$ is equal to the same product with the matrix $B^{L}$ of size $2^{L-1} \times\left(2^{L}-1\right)$.

Proof. The Theorem 1 is proved by a direct verification of the splitting scheme (11), (12). For example, we multiply the second row of the matrix $\left[P^{L} \mid Q^{L}\right]$ by the first three columns of the matrix $R^{L}$, constructed alike to the sample of matrices above:

$$
\begin{gathered}
\frac{11}{16} \cdot 12-1.35 \cdot 5-\frac{1}{2} \cdot 3=0, \frac{11}{16} \cdot 4+1.35 \cdot 5-\frac{1}{2} \cdot 1=9, \\
\frac{11}{16} \cdot(-108)+\frac{1}{8} \cdot 72+1.35 \cdot \frac{215}{3}-\frac{1}{2} \cdot 63=0 .
\end{gathered}
$$

These equalities mean that $\xi_{0}$ equals to $\frac{1}{9} C_{0}$ during the solution of the system (12) with the matrix $G^{L}$ obtained from the formula (11). The same manipulations with the first row of the matrix $\left[P^{L} \mid Q^{L}\right]$ give values

$$
\begin{gathered}
\frac{1}{4} \cdot 12+1 \cdot 5=8, \frac{1}{4} \cdot 4-1 \cdot 5=-4, \\
\frac{1}{4} \cdot(-108)-1 \cdot \frac{215}{3}=-\frac{296}{3} .
\end{gathered}
$$

Moving the value $-4 \xi_{0}=-\frac{4}{9} C_{0}$ to the right-hand side of the resulting equation, we obtain the first equation of the system with respect to the values $\Xi^{L}$ :

$$
8 \xi_{-1}-\frac{296}{3} \xi_{1}=C_{-1}+\frac{4}{9} C_{0} .
$$

Similar manipulations with the fourth row of the matrix $\left[P^{L} \mid Q^{L}\right]$ guarantee the justification that $\xi_{2}$ equals to $\frac{1}{2} C_{2}$ during the solution of the system (12) with the matrix $G^{L}$. The same manipulations with the third row of the matrix $\left[P^{L} \mid Q^{L}\right]$ give values

$$
\begin{gathered}
\frac{1}{2} \cdot 12+0.6 \cdot 5+1 \cdot 3=12, \frac{1}{2} \cdot 4-0.6 \cdot 5+1 \cdot 1=0, \\
\frac{1}{2} \cdot(-108)+\frac{1}{2} \cdot 72-0.6 \cdot \frac{215}{3}+1 \cdot 63=2, \\
\frac{1}{2} \cdot 4+1 \cdot 1=3, \frac{1}{2} \cdot 4+1 \cdot 1=3 .
\end{gathered}
$$

Moving the value $3 \xi_{2}=\frac{3}{2} C_{2}$ to the right-hand side of the resulting equation, we obtain the second equation of the system with respect to the values $\Xi^{L}$ :

$$
12 \xi_{-1}+2 \xi_{1}+3 \xi_{3}=C_{1}-\frac{3}{2} C_{2}
$$

and so on. For example, the following in order equations have the form:

$$
\begin{aligned}
54 \xi_{1}+10 \xi_{3}+3 \xi_{5} & =C_{3}-\frac{3}{2} C_{2}-\frac{3}{2} C_{4}, \\
3 \xi_{3}+10 \xi_{5}+3 \xi_{7} & =C_{5}-\frac{3}{2} C_{4}-\frac{3}{2} C_{6},
\end{aligned}
$$


The last equations are symmetric to the equations received above. The advantage of the adopted system, in contrast to the original system, is that it has only three non-empty diagonals. This allows us to apply the widely known method of elimination [20, p. 73] to its solution. Nevertheless, the stability of this method is not guaranteed, since the property of diagonal dominance is not satisfied in the first three and the last three equations. We multiply the first equation of the system by $(-3)$, and the second by 2 and we add. As a result, the unknown $\xi_{-1}$ is eliminated and the resulting equation takes the form

$$
300 \xi_{1}+6 \xi_{3}=2 C_{1}-3 C_{-1}-3 C_{2}-\frac{4}{3} C_{0} .
$$

We multiply the resulting equation by $(-9)$, and the third equation by 50 and add. As a result, the unknown $\xi_{1}$ is eliminated and the resulting equation takes the form $446 \xi_{3}+150 \xi_{5}=50 C_{3}-48 C_{2}-$ $75 C_{4}-18 C_{1}+27 C_{-1}+12 C_{0}$.

Similar transformations apply to the last three equations of the system. Thus, the conditions (13) and a) of the theorem can be written in the matrix form as the equation (12). At the same time, the system obtained has a strict diagonal dominance in all equations. Therefore, the elimination method is stable in application to the resulting system. The case b) follows immediately from the system (6) with $L=3$ after renaming the variables and permutation and rescaling of terms to receive the form with diagonal dominance. Hence, the conditions of Theorem 1 give a solution of the system (6).

The number of arithmetic operations needed to solve the system (14) by elimination is $3 \cdot\left(2^{L-1}-5\right)$ additions, $3 \cdot\left(2^{L-1}-5\right)$ multiplications, and $2 \cdot\left(2^{L-1}-\right.$ 5) +1 divisions [20, p. 76]. To calculate the right-hand sides of the equations, we need $3 \cdot\left(2^{L-1}-6\right)+6$ multiplications and $2 \cdot\left(2^{L-1}-6\right)+6$ additions; to obtain the spline coefficients at the nodes of a sparse grid, $3 \cdot\left(2^{L-1}-1\right)+18$ multiplications and $2 \cdot\left(2^{L-1}-1\right)+14$ additions is required. The most time-consuming part of the algorithm is the calculation of shifted wavelet coefficients: $5 \cdot\left(2^{L-1}-4\right)$ multiplications and $4 \cdot\left(2^{L-1}-4\right)$ additions. If we do not make any differences between arithmetic operations, then the total number of such operations for one step of the shifted wavelet decomposition is $27 \cdot 2^{L-1}-64$. Taking into account that $L=3$ in the last stage of thinning, we get the number of arithmetic operations to calculate the total set of shifted wavelet coefficients: $27 \cdot 2^{L}-64 L+20$. In comparison with the previously known fast algorithm [14] of the discrete wavelet transform, this algorithm makes it possible to obtain shifted wavelet decomposition coefficients in a different way with a comparable number of opera- tions. The advantages of the new algorithm are its stability property and the programmatic usability, since at each stage one system of linear equations (instead of two systems in the known algorithm) is solved with a matrix having a strict diagonal dominance.

\section{Recommendations for practical calculations}

In a real situation of wavelet analysis of a discrete signal, the homogeneous boundary conditions necessary for creation of a shifted wavelet decomposition are not satisfied. Therefore, before applying the above algorithm, it is necessary to subtract values of the cubic interpolation Hermite polynomial $f(a)+(x-a)\left[f^{\prime}(a)+t(B+t A)\right][21]$, where

$$
\begin{aligned}
A & =-2(f(b)-f(a)) /(b-a)+f^{\prime}(a)+f^{\prime}(b), \\
B & =-A+(f(b)-f(a)) /(b-a)-f^{\prime}(a), \\
t & =(x-a) /(b-a),
\end{aligned}
$$

from set values.

After shifted wavelet analysis of obtained differences and reconstruction on shifted wavelet coefficients of the approximating spline of the third degree, values of this polynomial are added to it.

Also, instead of original decomposition coefficients based on $B$-splines, you can use values of functions that differ little from them. For the case of wavelet data processing on a uniform grid, this trick is very popular in the literature around the world; it is called "Wavelet Crime" [22, p. 232], [23], [24].

\section{Conclusion}

A pioneering application of the author's procedure for the even-odd splitting of defining system of shifted wavelet decomposition onto the basis of $B$-splines is considered. The procedure for the case of Hermite shifted wavelets is adjusted to approximate functions that do not require the setting of derivatives values, which is important for practice. The advantage of the proposed procedure over other methods of compression is the ability to adaptively select the nodes of the approximating spline based on the coefficients of its shifted wavelet expansion. The extension of the proposed method to higher-order splines and higherorder zero moments can offer new possibilities for developing stable algorithms for constructing and applying spline-wavelets.

\section{References:}

[1] C.K. Chui, An Introduction to Wavelets, Academic Press, New York, London, 1992.

[2] M.W. Frazier, An Introduction to Wavelets through Linear Algebra, Springer-Verlag, New York, 1999. 
[3] M. Lyche, K. Mǿrken, E. Quak, Theory and algorithms for non-uniform spline wavelets, Multivariate Approximation and Applications, eds. N. Dyn, D. Leviatan, D. Levin, A. Pinkus, Cambridge University Press, Cambridge, 2001, pp. 152-187.

[4] K. Koro, K. Abe, Non-orthogonal spline wavelets for boundary element analysis, Engineering Analysis with Boundary Elements, Vol.25, 2001, pp. 149-164.

[5] I.Y. Novikov, M.A. Skopina, V.Y. Protasov, Wavelet Theory, Translations of Mathematical Monographs, Vol. 239, American Mathematical Society, Providence (RI), 2011.

[6] M. Jansen, Non-equispaced B-spline wavelets, International Journal of Wavelets, Multiresolution and Information Processing, Vol.14, No.6, 2016, 1650056.

[7] D. Ĉerná, V. Finêk, Cubic spline wavelets with complementary boundary conditions, Applied Mathematics and Computation, Vol.219, 2012, pp. 1853-1865.

[8] B. Han, Z. Shen, Wavelets with Short Support, SIAM Journal on Mathematical Analysis, Vol.38, No.2, 2006, pp. 530-556.

[9] D. Cerná, V. Finêk, Cubic spline wavelets with short support for fourth-order problems, Applied Mathematics and Computation, Vol.243, 2014, pp. 44-56.

[10] B.M. Shumilov, Multiwavelets of the thirddegree hermitian splines orthogonal to cubic polynomials, Mathematical Models and Computer Simulations, Vol.5, No.6, 2013, pp. 511519.

[11] B.M. Shumilov, On multiwavelets of Hermite splines of odd degree, Siberian Electronic Mathematical Reports, Vol.12, 2015, pp. 138-147.

[12] B.M. Shumilov, Displaced Multiwavelets and Splitting Algorithms, Advanced Engineering Materials and Modeling, eds. A. Tiwari, N.A. Murugan, R. Ahuja, John Wiley \& Sons, Ltd, Hoboken, NJ, USA, 2016, pp. 435-494.

[13] F. Aràndiga, A. Baeza, R. Donat, Discrete multiresolution based on hermite interpolation: computing derivatives, Communications in Nonlinear Science and Numerical Simulation, Vol.9, 2004, pp. 263-273.
[14] J. Wang, Cubic spline wavelet bases of sobolev spaces and multilevel interpolation, Applied and Computational Harmonic Analysis, Vol.3, No.2, 1996, pp. 154-163.

[15] B.M. Shumilov, Semi-orthogonal splinewavelets with derivatives and the algorithm with splitting, Numerical Analysis and Applications, Vol.10, No.1, 2017, pp. 90-100.

[16] C. De Boor, A Practical Guide to Splines, Applied Mathematical Sciences, Vol.27, SpringerVerlag, New York, 1978.

[17] E.J. Stollnitz, T.D. DeRose, D.H. Salesin, Wavelets for Computer Graphics, The Morgan Kaufmann Series in Computer Graphics, 1996.

[18] B.M. Shumilov, S.M. Matanov, Supercompact cubic multiwavelets and algorithm with splitting, 2011 International Conference on Multimedia Technology, 2011, pp. 2636-2639.

[19] B.M. Shumilov, Cubic multiwavelets orthogonal to polynomials and a splitting algorithm, $\mathrm{Nu}$ merical Analysis and Applications, Vol.6, No.3, 2013, pp. 247-259.

[20] A.A. Samarskii, E.S. Nikolaev, The Elimination Method, Numerical Methods for Grid Equations, Birkhauser Basel, 1989, pp. 61-115.

[21] Z.M. Sulaimanov, B.M. Shumilov, A splitting algorithm for the wavelet transform of cubic splines on a nonuniform grid, Computational Mathematics and Mathematical Physics, Vol.57, No.10, 2017, pp. 1577-1591.

[22] G. Strang, T. Nguyen, Wavelets and filter banks, Cambridge Press, Wellesley, 1996.

[23] P. Qian, B.A. Francis, Solution of a Wavelet Crime, Topics in Control and its Applications: A Tribute to Edward J. Davison, Springer London, London, 1999, pp. 143-156.

[24] W. Rakowski, Prefiltering in Wavelet Analysis Applying Cubic B-Splines, International Journal of Electronics and Telecommunications, Vol.60, No.4, 2014, pp. 331-340.

\section{Sources of funding for research presented in a scientific article or scientific article itself}

Russian Foundation for Basic Research (RFBR) 


\section{Creative Commons Attribution License 4.0} (Attribution 4.0 International, CC BY 4.0)

This article is published under the terms of the Creative Commons Attribution License 4.0

https://creativecommons.org/licenses/by/4.0/deed.en_US 